\title{
Avaliação cefalométrica das alterações verticais e ântero-posteriores em pacientes Classe II esquelética, tratados com aparelho extrabucal de tração cervical ou combinada
}

Márlio Vinícius de Oliveira*, Luiz Antônio Alves Bernardes**

\section{Resumo}

Objetivo: avaliar cefalometricamente as alterações ântero-posteriores e verticais em pacientes Classe II esquelética (ANB $\geq 5^{\circ}$ ), tratados com aparelho extrabucal cervical (grupo 1) associado a aparelho fixo do tipo Edgewise ou tratados com aparelho extrabucal de tração combinada (grupo 2) associado ao mesmo. Metodologia: a amostra consistiu-se de 60 radiografias cefalométricas laterais obtidas nas fases pré-tratamento e pós-tratamento de 30 indivíduos leucodermas, sendo 13 do gênero masculino e 17 do feminino. A idade média dos 15 pacientes do grupo 1, no pré-tratamento, era de 10 anos e 7 meses, e no pós-tratamento era de 13 anos e 9 meses. Os 15 pacientes do grupo 2 apresentavam idade média, no pré-tratamento, de 11 anos e 5 meses e no pós-tratamento a idade média era de 14 anos e 9 meses. As medidas cefalométricas iniciais e finais foram analisadas e comparadas pelo teste $t$ de Student. Resultados e Conclusões: não houve alteração significante no padrão de crescimento facial durante o tratamento em nenhum dos grupos avaliados. Nos pacientes do grupo 2, que possuíam tendência de crescimento vertical (GoGn-SN>36 ${ }^{\circ}$ ), o aparelho extrabucal de tração combinada, mesmo não provocando efeito extrusivo sobre os molares superiores, não foi capaz de diminuir o ângulo do plano mandibular. A maxila apresentou uma restrição no seu deslocamento anterior e verticalmente manteve-se estável. A mandíbula expressou seu crescimento e deslocou-se anteriormente, porém manteve sua inclinação inalterada. A relação maxilomandibular apresentou uma melhora significante com redução sensível do ANB.

Palavras-chave: Classe II esquelética. Aparelhos extrabucais cervical e combinado. Aparelho ortodôntico fixo.

\section{INTRODUÇÃO}

O crescimento dos ossos maxilares influencia diretamente o perfil e a harmonia da face. Caso a maxila e a mandíbula não estejam bem relacionadas entre si, o tratamento ortodôntico realizado no período do surto de crescimento poderá reposicionar e equilibrar a relação das mesmas. Os aparelhos extrabucais são os mais utilizados na Ortodontia para a correção desses mal relacionamentos. O tratamento da Classe II com esses aparelhos produz alterações no esqueleto crânio facial, porém permanecem controvérsias em re-

\footnotetext{
* Especialista em Ortodontia pela Universidade Federal de Alfenas - UNIFAL.

** Mestre em Ortodontia pela Universidade Federal do Rio de Janeiro-UFRJ. Professor convidado do Curso de Especialização em Ortodontia da Universidade Federal de Alfenas - UNIFAL. Diplomado pelo Board Brasileiro de Ortodontia e Ortopedia Facial.
} 
lação à magnitude e direção dessas alterações ${ }^{1}$.

A má oclusão de Classe II, $1^{\text {a }}$ divisão de Angle, está presente em $40 \%$ da população que procura tratamento ortodôntico ${ }^{2}$. Esse tipo de problema apresenta uma etiologia multifatorial e, consequentemente, mais de uma possibilidade de tratamento. $\mathrm{O}$ aparelho extrabucal cervical tem sido usado, mais freqüentemente, em casos de dimensão vertical reduzida e protrusão esquelética da maxila, produzindo deslocamento distal da mesma e aumento da dimensão vertical, devido à extrusão dos molares, gerando rotação da mandíbula no sentido horário ${ }^{3}$.

O extrabucal de tração combinada produz somente o movimento distal dos molares, desse modo vem sendo utilizado em pacientes com padrão de crescimento vertical. Nesses casos, o efeito extrusivo sobre os molares superiores produzido pelo aparelho extrabucal cervical poderia aumentar ainda mais a dimensão vertical, dificultando a correção da Classe II. Estudos têm mostrado que o principal efeito negativo do aparelho extrabucal cervical é a rotação da mandíbula para baixo e para trás, abrindo a mordida e aumentando o ângulo do plano mandibular ${ }^{4,5}$.

O movimento da maxila ou do primeiro molar depende da direção da linha de ação da força aplicada pelo extrabucal e da sua relação com o centro de resistência do dente, da maxila e do complexo dentoalveolar maxilar, ${ }^{6,7,8}$. Portanto, o principal fator na escolha clínica entre esses aparelhos é a preocupação em relação à possibilidade de produzir alterações indesejáveis no plano mandibular, como conseqüência da orientação da força aplicada ${ }^{9}$.

O objetivo deste trabalho foi avaliar o comportamento da maxila e mandíbula nos sentidos ântero-posterior e vertical, em pacientes com padrão esquelético de Classe II (ANB > 5), tratados com aparelho extrabucal de tração cervical associado ao aparelho fixo Edgewise e extrabucal de tração combinada associado ao mesmo tipo de aparelho fixo.

\section{MATERIAL E MÉTODOS}

O material utilizado nesta pesquisa constitui-se de 60 telerradiografias de perfil, pré-tratamento e pós-tratamento, de 30 indivíduos leucodermas, 13 do gênero masculino e 17 do gênero feminino, portadores de má oclusão Classe II esquelética, tratados pelo mesmo ortodontista, sendo 15 indivíduos tratados com extrabucal de tração cervical associado ao aparelho fixo Edgewise (grupo 1) e os outros 15 com extrabucal de tração combinada associado ao mesmo tipo de aparelho fixo (grupo 2).

A amostra foi selecionada de uma clínica privada de um dos autores em Poços de Caldas, Minas Gerais, Brasil, e os pacientes foram tratados no período entre 1989 e 2004. Foram utilizados os seguintes critérios para selecioná-los: portadores de Classe II esquelética com $\mathrm{ANB} \geq 5^{\circ}$, tratamento iniciado antes do surto de crescimento puberal, pacientes do grupo 1 possuíam o ângulo GoGn_Sn $\leq 36^{\circ}$, pacientes do grupo 2 possuíam o ângulo GoGn_Sn > $36^{\circ}$. Todos os pacientes foram tratados sem extrações dentárias.

A idade média dos pacientes do grupo 1, no pré-tratamento, era de 10 anos e 7 meses, com idade mínima de 9 anos e máxima de 13 anos. Já a idade média no pós-tratamento foi de 13 anos e 9 meses, com idade mínima de 13 anos e 2 meses e máxima de 15 anos e 3 meses. $O$ tempo médio de tratamento foi de 3 anos e 2 meses.

Os pacientes do grupo 2 tinham idade média, no pré-tratamento, de 11 anos e 5 meses, com idade mínima de 9 anos e 3 meses e máxima de 13 anos e 2 meses. No pós-tratamento a idade média era de 14 anos e 9 meses, com idade mínima de 11 anos e máxima de 18 anos. $\mathrm{O}$ tempo médio de tratamento desse grupo foi de 3 anos e 4 meses.

Para a obtenção das radiografias cefalométricas de perfil os pacientes foram posicionados com o plano de Frankfurt paralelo ao plano horizontal (solo), os dentes em oclusão cêntrica e os lábios em repouso.

O traçado cefalométrico foi efetuado em folha de acetato transparente Ultraphan (3M Unitek, 
Monrovia, USA), tamanho 20,3 x 25,4cm e espessura de 0,003", em negatoscópio (VH, Araraquara, Brasil), com lapiseira Pentel carregada com grafite preto, P3, 0,5mm de espessura (P203, Pentel, Brasil), template com régua milimetrada (Tracing Template, 3M Unitek, Monrovia, USA) e transferidor com graduação em milímetros e graus (Protractor, 3M Unitek, Monrovia, USA). No caso de estruturas bilaterais (ângulo goníaco, dentes, key ridge, órbitas, etc.), não havendo sobreposição, considerou-se a média de ambos os lados.

Todos os traçados foram realizados pelo mesmo pesquisador e, tendo em vista a necessidade de avaliar a reprodutibilidade da pesquisa na determinação dos pontos cefalométricos, foram realizados ensaios preliminares com a finalidade de verificar o erro no método empregado. Desse modo, 10 telerradiografias, escolhidas de modo aleatório, foram traçadas em intervalos pré-determinados (no mínimo duas semanas), entre o primeiro e o segundo traçado. $\mathrm{O}$ maior erro encontrado foi de $0,5^{\circ}$ e o menor $0,1^{\circ}$.

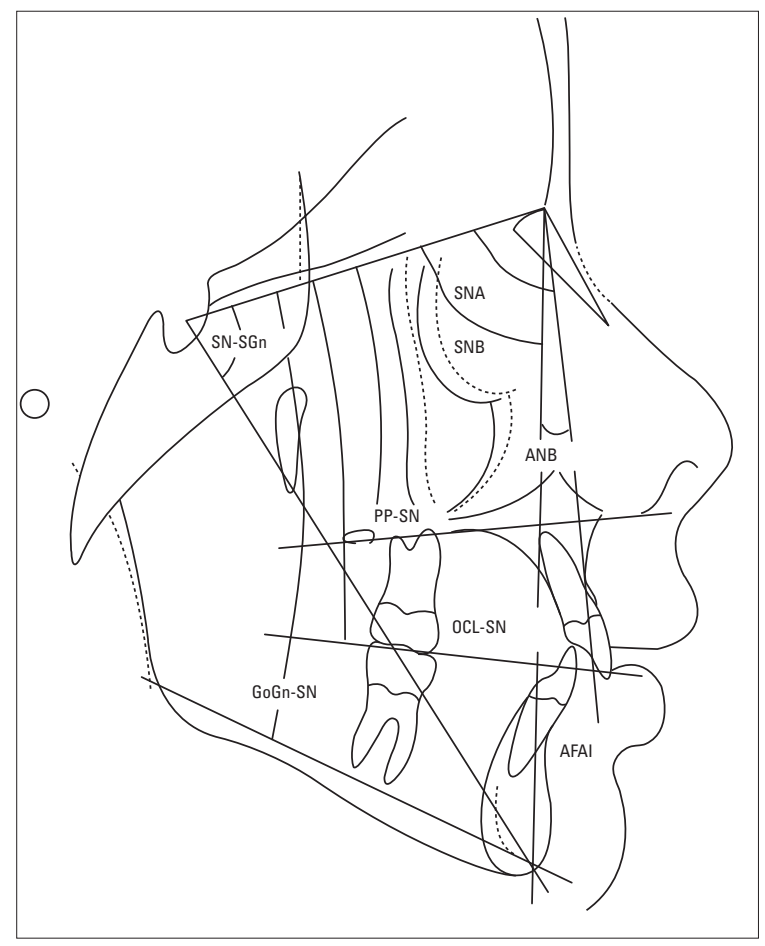

FIGURA 1 - Representação das medidas angulares e medida linear (AFAI) utilizadas nesse estudo.
As estrutura anatômicas que compõem o traçado cefalométrico foram descritas por Vilella ${ }^{10}$ e são utilizadas como padrão no Curso de Especialização em Ortodontia da UNIFAL. Após a obtenção do desenho anatômico, foram marcados os seguintes pontos cefalométricos: sela, násio, espinha nasal anterior, espinha nasal posterior, subespinhal, supramental, gnátio, mento e gônio). Com os pontos cefalométricos citados anteriormente, formaram-se linhas e planos, e a partir daí foram obtidas as seguintes medidas angulares: 1) SNA, SNB e ANB, que fornece o posicionamento ântero-posterior maxilomandibular e 2) GoGn-SN, SN-SGn, OCL$\mathrm{SN}$ e PP-SN, mostrando as alterações verticais. Foi feita também a medida linear AFAI (Fig. 1).

No grupo 1, o aparelho com arco facial utilizado possuía um arco interno soldado a um arco externo na região dos incisivos, com diâmetro de 0,051 " e 0,071", respectivamente (Morelli, Sorocaba, Brasil). Uma tala cervical com elástico (Morelli, Sorocaba, Brasil) aplicava uma força de 500g, que era

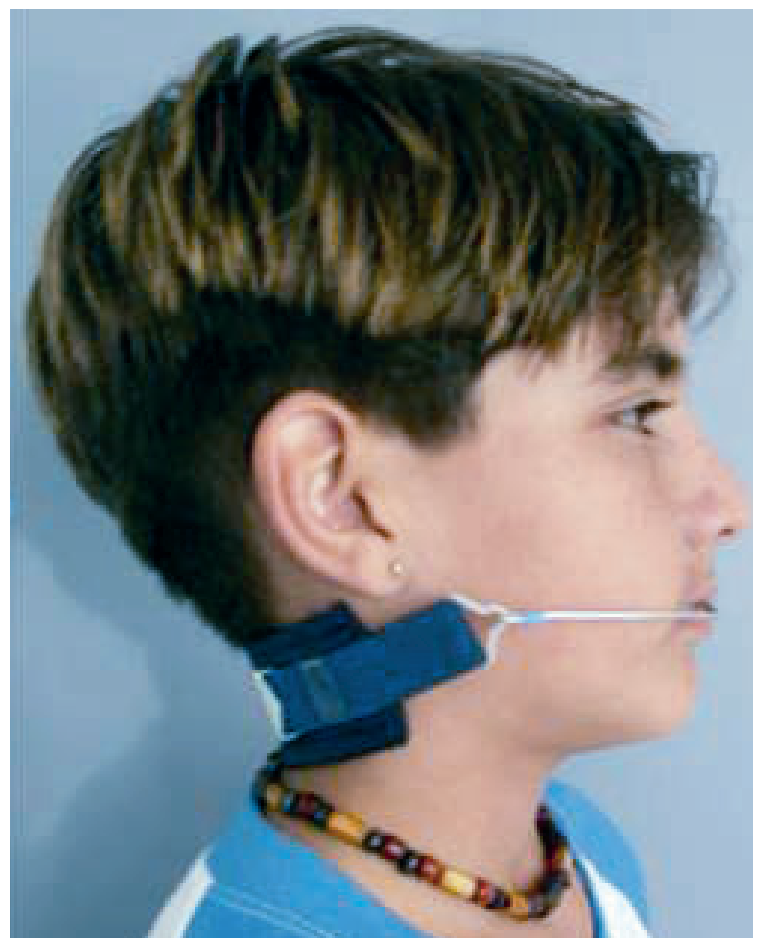

FIGURA 2 - Adaptação da tala cervical ao arco externo do aparelho. 
verificada mensalmente, quando encaixada ao arco externo do aparelho (Fig. 2). Durante o tratamento, se houvesse necessidade, ajustes no arco externo do aparelho poderiam ser feitos para permitir que a força sempre atuasse sobre o centro de resistência do molar superior, desse modo este dente não sofria nenhuma inclinação durante o movimento de distalização. Os pacientes foram orientados a utilizarem o aparelho 14 horas/dia.

O aparelho extrabucal utilizado nos pacientes do grupo 2 possuía um arco externo soldado a um arco interno (Morelli, Sorocaba, Brasil), com as mesmas dimensões do aparelho citado anteriormente. No entanto, foi soldado um gancho no arco externo para a aplicação da tração alta. Uma tala cervical com elástico (Morelli, Sorocaba, Brasil) era acoplada à extremidade distal do arco externo e aplicava uma força de 250g; um casquete parietal com elástico (Morelli, Sorocaba, Brasil) foi acoplado ao gancho soldado e também aplicava uma força de 250g (Fig. 3). O aparelho era ajustado de modo que a linha de ação da força resultante ficasse para-

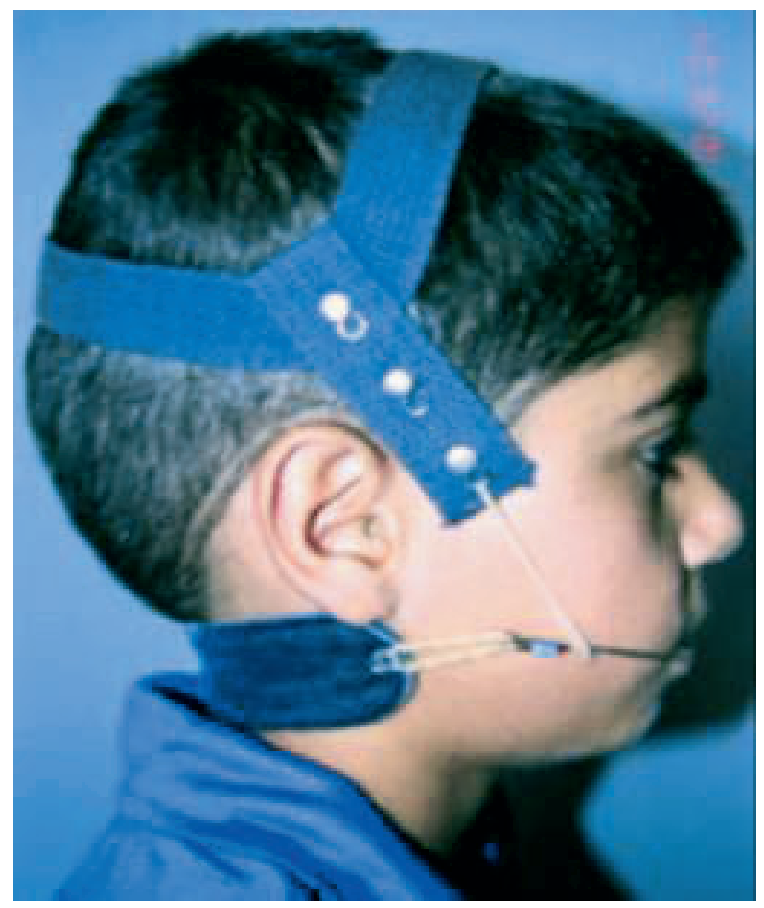

FIGURA 3 - Adaptação das talas cervical e parietal ao arco externo do aparelho. lela ao plano oclusal e coincidisse com o centro de resistência dos primeiros molares superiores.

Nenhuma avaliação quanto à cooperação do paciente no uso dos aparelhos extrabucais foi considerada, porém, entende-se que tais pacientes eram bons colaboradores, uma vez que em todos os casos a relação ântero-posterior entre maxila e mandíbula foi corrigida.

\section{Análise estatística}

A análise estatística desenvolvida compreendeu dois momentos diferentes. Em uma primeira análise foi realizada a comparação dos dois grupos aleatórios e independentes e em um segundo estágio foi realizada a análise intragrupo com o objetivo de verificar as variações de um mesmo indivíduo nas fases inicial e final dos tratamentos.

As alterações médias das grandezas cefalométricas entre as fases inicial e final dos pacientes foram analisadas com o teste $t$ de Student com o nível de significância de 5\%.

\section{RESULTADOS}

As tabelas 1 e 2 mostram as diferenças entre os tempos inicial e final do tratamento ortodôntico nos pacientes dos grupos 1 e 2 , respectivamente. A análise estatística realizada permitiu verificar que ambos os grupos apresentaram redução no valor médio dos ângulos SNA e ANB; aumento médio do ângulo SNB e da medida linear AFAI. No grupo 1 houve ainda redução média do ângulo OCL-SN. As alterações nos ângulos GoGn-SN e SN-SGn não foram significativas em nenhum dos dois grupos avaliados.

A tabela 3 e o gráfico 1 apresentam os resultados da comparação das alterações cefalométricas médias ocorridas nos pacientes dos grupos 1 e 2, durante o tratamento. A aplicação do teste $t$ de Student mostrou que não houve alteração estatisticamente significante na comparação das grandezas cefalométricas, comprovando que as alterações médias dos resultados do tratamento com os dois tipos de aparelhos foram semelhantes, levando-se em 
Tabela 1 - Comparação entre as alterações médias ocorridas durante o tratamento dos 15 pacientes do grupo 1.

\begin{tabular}{|c|c|c|c|c|c|c|c|}
\hline \multirow{2}{*}{ medida } & \multicolumn{2}{|c|}{ inicial } & \multicolumn{2}{|c|}{ final } & \multirow{2}{*}{$\begin{array}{l}\text { dif } \\
x\end{array}$} & \multirow{2}{*}{$\begin{array}{c}\text { teste } t \\
\mathbf{p}\end{array}$} & \multirow{2}{*}{ sig. } \\
\hline & $\mathrm{x}$ & d.p. & $\mathrm{x}$ & d.p. & & & \\
\hline SNA & 81,45 & 3,13 & 79,80 & 3,30 & $-1,65$ & 0,039 & s. \\
\hline SNB & 75,80 & 3,90 & 76,65 & 4,80 & 0,85 & 0,041 & s. \\
\hline ANB & 5,90 & 1,40 & 3,50 & 1,60 & $-2,40$ & 0,016 & s. \\
\hline SN-SGn & 68 & 3,20 & 68,05 & 2,60 & 0,05 & 0,237 & n.s. \\
\hline GoGn-SN & 30,70 & 2,50 & 30,50 & 4,10 & 0,20 & 0,373 & n.s. \\
\hline OCL-SN & 18,25 & 4,60 & 17,25 & 2,16 & $-1,00$ & 0,031 & s. \\
\hline PP-SN & 9,15 & 2,90 & 9,35 & 3,03 & 0,20 & 0,078 & n.s. \\
\hline AFAI & 62,05 & 3,13 & 67 & 3,70 & 4,95 & 0,024 & s. \\
\hline
\end{tabular}

S.: significante para $p<0,05$, n.s.: não significante.

Tabela 2 - Comparação entre as alterações médias ocorridas durante o tratamento dos 15 pacientes do grupo 2.

\begin{tabular}{|c|c|c|c|c|c|c|c|}
\hline \multirow{2}{*}{ medida } & \multicolumn{2}{|c|}{ inicial } & \multicolumn{2}{|c|}{ final } & \multirow{2}{*}{$\begin{array}{l}\text { dif } \\
x\end{array}$} & \multirow{2}{*}{$\begin{array}{c}\text { teste } t \\
\text { p }\end{array}$} & \multirow{2}{*}{ sig. } \\
\hline & $\mathrm{x}$ & d.p. & $\mathrm{x}$ & d.p. & & & \\
\hline SNA & 80,60 & 2,79 & 78,80 & 2,52 & $-1,80$ & 0,029 & s. \\
\hline SNB & 74,30 & 2,05 & 75,70 & 1,25 & 1,40 & 0,027 & s. \\
\hline ANB & 6,30 & 1,41 & 3,10 & 1,44 & $-3,20$ & 0,014 & s. \\
\hline SN-SGn & 69,70 & 1,94 & 70,10 & 2.42 & 0,40 & 0,291 & n.s. \\
\hline GoGn-SN & 38,20 & 3,67 & 37,44 & 4,30 & $-0,80$ & 0,098 & n.s. \\
\hline OCL-SN & 19,40 & 3,02 & 19,00 & 3,16 & $-0,40$ & 0,371 & n.s. \\
\hline PP-SN & 8,70 & 2,70 & 8,90 & 1,34 & 0,20 & 0,078 & n.s. \\
\hline AFAI & 64,90 & 4,81 & 71,10 & 6,91 & 6,20 & 0,047 & s. \\
\hline
\end{tabular}

s.: significante para $p<0,05$, n.s.: não significante.

Tabela 3 - Comparação entre as alterações médias ocorridas nos pacientes dos Grupos 1 e 2 durante 0 tratamento.

\begin{tabular}{|c|c|c|c|c|c|c|c|}
\hline \multirow{2}{*}{ medida } & \multicolumn{2}{|c|}{ grupo 1} & \multicolumn{2}{|c|}{ grupo 2} & \multicolumn{2}{|c|}{ teste $\mathbf{t}$} & \multirow{2}{*}{ sig. } \\
\hline & $\mathbf{x}$ & d.p. & $\mathbf{x}$ & d.p. & t & $\mathbf{p}$ & \\
\hline SNA & $-1,65$ & 1,64 & $-1,80$ & 2,15 & $-1,443$ & 0,246 & n.s. \\
\hline SNB & 0,80 & 1,15 & 1,40 & 1,43 & 2,128 & 0,118 & n.s. \\
\hline ANB & $-2,40$ & 1,32 & $-3,20$ & 1,69 & $-0,958$ & 0,326 & n.s. \\
\hline SN-SGn & 0,07 & 1,44 & 0,40 & 1,58 & 1,052 & 0.337 & n.s. \\
\hline GoGn-SN & 0,00 & 1,93 & $-0,44$ & 2,40 & 1,268 & 0,281 & n.s. \\
\hline OCL-SN & $-1,00$ & 2,83 & $-0,40$ & 2,39 & 1,989 & 0,103 & n.s. \\
\hline PP-SN & 0,33 & 1,50 & 0,10 & 1,91 & 1,633 & 0,227 & n.s. \\
\hline AFAI & 4,80 & 1,82 & 6,20 & 3,55 & 0,806 & 0,459 & n.s. \\
\hline
\end{tabular}

s.: significante para $p<0,05$, n.s.: não significante. 


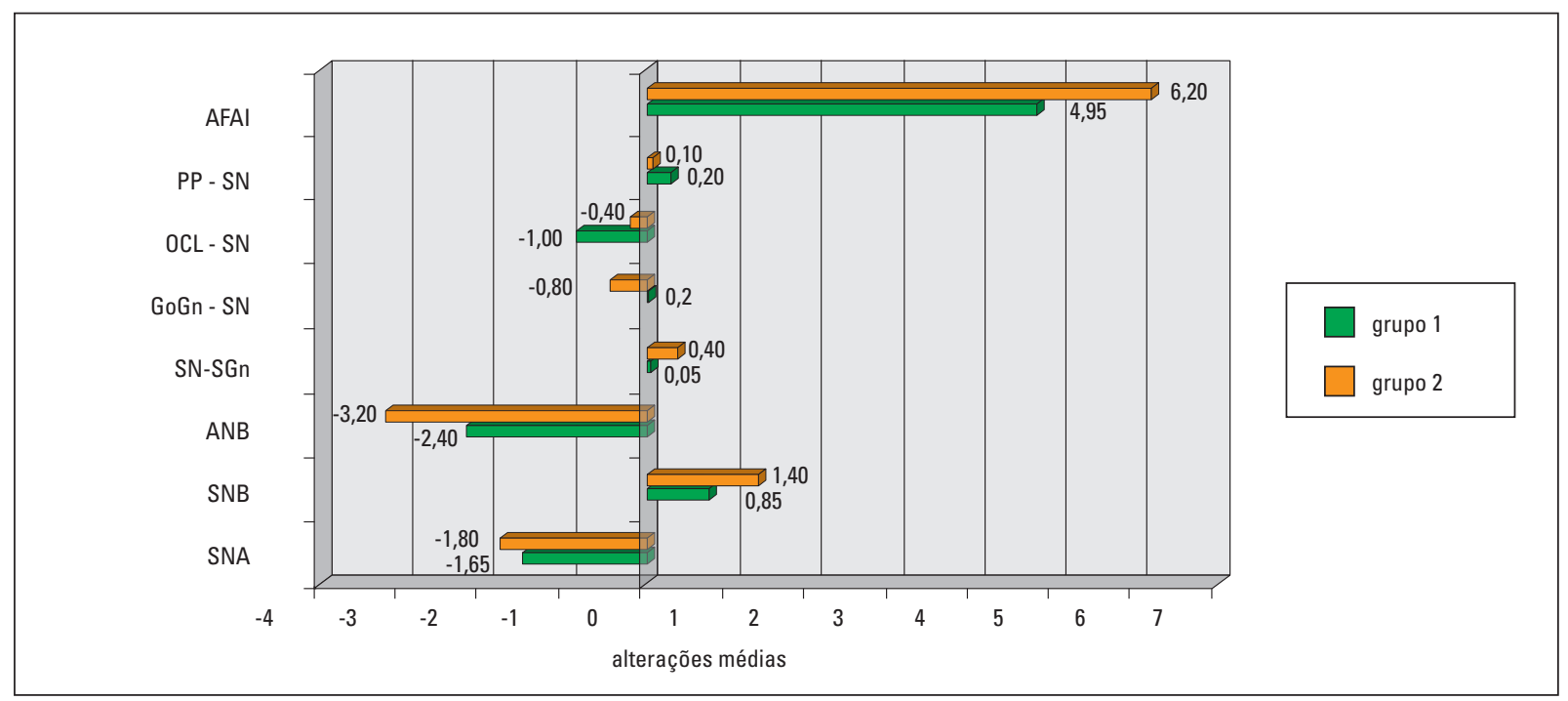

GRÁFICO 1 - Alterações médias das grandezas pesquisadas nos grupos 1 e 2.

consideração que os pacientes do grupo 2 tinham padrão de crescimento vertical.

\section{DISCUSSÃO}

Avaliando as características da má oclusão de Classe II esquelética, constatou-se que há muita controvérsia entre os autores. Vários fatores esqueléticos, dentários e funcionais interagem para a conformação da relação distal do arco dentário inferior com o superior. Segundo alguns autores, poderá estar presente uma retroposição mandibular ${ }^{11,12}$, protrusão maxilar ${ }^{13,14}$ ou uma combinação desses dois fatores ${ }^{15,16,17,18}$.

Nesse estudo, os pacientes de ambos os grupos apresentavam a maxila bem posicionada, de acordo com os critérios de avaliação propostos por Steiner ${ }^{19}$. O valor médio do ângulo SNA, no início do tratamento, era $81,4^{\circ}$ para o grupo 1 e $80,6^{\circ}$ para o grupo 2. A mandíbula, no entanto, apresentava-se retruída em ambos os grupos. $\mathrm{O}$ valor médio do ângulo SNB, no início do tratamento, era de $75,8^{\circ}$ para o grupo 1 e $74,3^{\circ}$ para o grupo 2 .

Quanto ao padrão de crescimento dos pacientes Classe II esquelética, a maioria dos autores concorda que há uma predominância do componente vertical $^{20,21,22,23,24}$.
$\mathrm{Na}$ amostra desse estudo, em relação ao padrão de crescimento, todos os pacientes do grupo 1 apresentavam o ângulo GoGn-SN $\leq 36^{\circ}$ e os do grupo 2 apresentavam o ângulo GoGn-SN $>36^{\circ}$. Portanto, os pacientes do grupo 2 tinham tendência de crescimento vertical.

Existem vários tipos de aparelhos extrabucais para retrair a maxila no tratamento da má oclusão de Classe II, no entanto, o principal fator na escolha clínica entre esses aparelhos é a preocupação em relação à possibilidade de produzir alterações verticais indesejáveis, como rotação horária da mandíbula, por exemplo, em conseqüência da orientação da força aplicada.

Muitos pesquisadores têm afirmado que a mandíbula gira para trás e que o ângulo do plano mandibular aumenta com o uso da tração cervical ${ }^{4,5}$. Todavia, outros autores não observaram alteração do ângulo do plano mandibular decorrente dessa terapia ${ }^{3,25}$.

O ângulo GoGn-SN, nos pacientes do grupo 1, apresentou redução média de $0,2^{\circ}$ do início para o final do tratamento. Os resultados do teste $t$ de Student mostraram que essa redução não foi significante $(p>0,05)$. Estes dados indicam que o aparelho extrabucal cervical não alterou a inclinação do plano 
mandibular durante o tratamento, e estão de acordo com os achados de Hubbard et al..$^{25}$ e Lima ${ }^{3}$.

Nos pacientes do grupo 1, o que permitiu a manutenção do ângulo do plano mandibular do início para o final do tratamento pode ter sido a quantidade de crescimento condilar, que compensou a extrusão ou crescimento alveolar na região dos molares, conforme os achados de Schudy 22 .

Nos pacientes do grupo 2, o ângulo GoGn-SN apresentou redução média de $0,8^{\circ}$ do início para o final do tratamento. Apesar dos resultados do teste $t$ de Student mostrarem que essa redução não é significante ao nível de 95\% ( $p>0,05)$, acredita-se que o aparelho extrabucal de tração combinada controlou, relativamente bem, a extrusão dos primeiros molares superiores durante a correção da má oclusão de Classe II, visto que os pacientes desse grupo possuíam tendência de crescimento vertical. De acordo com Ricketts ${ }^{26}$, os padrões de crescimento estão bem estabelecidos, ou seja, ângulos do plano mandibular menores tendem a diminuir e os maiores a se manterem ou aumentarem. Portanto, os pacientes do grupo 2 possuíam tendência de aumento no ângulo do plano mandibular com o crescimento normal.

Quanto ao ângulo PP-SN, o tratamento da Classe II com extrabucal cervical tem proporcionado resultados onde verifica-se o aumento deste. Este ângulo apresentou um aumento nos trabalhos de O'Reilly et al. ${ }^{5}$ e Gandini Junior et al. ${ }^{27}$ Em acordo com estes trabalhos, a inclinação do plano palatino comportou-se de modo semelhante nos dois grupos avaliados, apresentando um aumento do ângulo PP-SN. Em ambos os grupos houve aumento médio de $0,2^{\circ}$ do início para o final do tratamento. Os resultados do teste $t$ de Student mostraram que esse aumento não é significante ( $p>0,05)$.

O tratamento da Classe II com a utilização de aparelho extrabucal apresentou um aumento da altura facial ântero-inferior (AFAI) em grande parte das pesquisas presentes na literatura, independente da fase da dentadura que o paciente se encon$\operatorname{tra}^{28,29,30}$. Cook et al. ${ }^{31}$, ao compararem este tratamento, na fase da dentadura mista, com um grupo de jovens com má oclusão de Classe II não tratados, verificaram que o aumento da AFAI ocorrido durante o tratamento foi inferior ao observado nos jovens que não receberam tratamento, indicando que o aumento da dimensão inferior da face apresentase diretamente relacionado ao crescimento natural. Neste trabalho verificou-se que a AFAI apresentou um aumento significante entre o início e o final do tratamento em ambos os grupos avaliados.

A AFAI aumentou em média 4,95mm do início para o final do tratamento, no grupo 1 . No grupo 2, este aumento foi em média de $6,20 \mathrm{~mm}$. Portanto, a comparação das alterações médias verificadas para os dois grupos mostraram semelhança do ponto de vista estatístico $(\mathrm{p}=0,459)$, apesar do aumento da AFAI ter ocorrido de maneira mais intensa no grupo 2, onde os pacientes apresentavam um padrão de crescimento vertical. Estes achados estão de acordo com Burke e Jacobson ${ }^{32}$, que avaliaram dois grupos de pacientes Classe II, $1^{\text {a }}$ divisão, com ângulos do plano mandibular elevados, tratados com tração cervical e parietal, e comprovaram que, após o tratamento, não houve diferenças significantes na altura da face ou no ângulo do plano mandibular. No entanto, Ricketts $^{26}$ discordou desses achados em seus trabalhos, nos quais comparou grupos de pacientes Classe I e II, tratados e não tratados, e verificou que certas mecânicas, como a tração cervical e os elásticos intermaxilares, provocam abertura da mordida e aumentam o comprimento da face.

Durante esta investigação, o aumento médio do ângulo SN-SGn foi de $0,05^{\circ}$ para o grupo le $0,4^{\circ}$ para o grupo 2. Tais alterações não foram estatisticamente significantes ao nível de 95\% ( $p>0,05)$. Estes resultados demonstram que nenhum dos tratamentos instituídos foi capaz de alterar significantemente o padrão de crescimento facial e confirmam os valores encontrados para o ângulo GoGn-SN (plano mandibular), que também não alterou significantemente durante o tratamento em nenhum dos dois grupos estudados. $\mathrm{O}$ estudo de Klein $^{33}$ discorda desses resultados e relacionou di- 
retamente a extrusão dos molares superiores com a rotação posterior da mandíbula e conseqüente abertura do eixo $Y$.

De acordo com Stöckli e Teuscher ${ }^{34}$, em um padrão típico de crescimento médio com deslocamento da sínfise para baixo e para frente ao longo do eixo Y, o declive da fossa mandibular e o crescimento vertical dos côndilos equilibram o movimento vertical para baixo do corpo maxilar e do processo alveolar superior mais o movimento para cima do processo alveolar inferior. Talvez isso justifique a manutenção do padrão de crescimento mesmo nos pacientes do grupo 1, nos quais a linha de ação da força aplicada pelo extrabucal exerce um efeito extrusivo sobre os primeiros molares superiores.

Uma outra medida vertical avaliada nesse estudo foi o ângulo entre o plano oclusal e a linha SN. Em ambos os grupos avaliados houve uma redução média no valor desse ângulo. No grupo 1 , a redução média foi de $1^{\circ}$, no grupo 2 foi de $0,40^{\circ}$. Esses valores não apresentaram significância estatística $(p>0,05)$.

Os aparelhos extrabucais provocam restrição do deslocamento anterior da maxila ${ }^{1,35,36,37}$, principalmente quando indicados na fase da dentadura mista, durante o surto de crescimento puberal ${ }^{38}$.

Nesse estudo, o comportamento do ângulo SNA durante o tratamento, foi estatisticamente semelhante para os dois grupos analisados. No grupo 1, a redução média do SNA foi de $1,65^{\circ}$. No grupo 2 , o SNA reduziu-se, em média, 1,8 $8^{\circ}$. Esses resultados mostram que houve uma restrição estatisticamente significante $(p<0,05)$ no deslocamento anterior $d a$ maxila, em ambos os grupos, durante o tratamento com aparelho extrabucal. Apesar dessa redução ter sido um pouco mais acentuada no grupo 2, a comparação entre os valores médios dos dois grupos não apresentou significância estatística $(\mathrm{p}=0,246)$.

A maioria dos trabalhos encontrados na literatura está de acordo com os resultados descritos acima. Vários autores observaram os efeitos do aparelho extrabucal no crescimento maxilar, sendo a retração do ponto A e a diminuição do ângulo SNA verificadas por Klein ${ }^{33}$, Mays ${ }^{4}$, Telles ${ }^{39}$, Cangialosi et al. ${ }^{40}$, Knight ${ }^{41}$, Henriques ${ }^{42}$ e Hubbard et al..$^{25}$

$K$ loehn $^{35}$ e Brodie $^{36}$ verificaram que, no crescimento normal, o processo alveolar e os dentes deslocam-se para frente e, se o ortodontista pudesse interromper esse movimento para frente em pacientes Classe II, a mandíbula poderia expressar seu crescimento normal, até atingir uma relação favorável com a maxila.

Os resultados obtidos na presente investigação, em relação ao posicionamento mandibular, estão de acordo com a opinião dos autores citados acima. No grupo 1, o aumento médio do ângulo SNB foi de $0,85^{\circ}$. O ângulo SNB no grupo 2 aumentou, em média, $1,40^{\circ}$. Esses resultados denotam um posicionamento anterior da mandíbula ao final do tratamento e são estatisticamente significantes ao nível de $95 \%(\mathrm{p}<0,05)$. Apesar dos jovens com padrão de crescimento vertical (grupo 2) terem apresentado um deslocamento anterior mandibular mais acentuado, quando comparamos as alterações médias nos dois grupos, não foi observada diferença significante entre eles $(p=0,118)$.

A redução do ângulo ANB e a conseqüente melhora no relacionamento maxilomandibular tem sido relatada quando do tratamento da má oclusão de Classe II com utilização de aparelhos extrabucais, durante o surto de crescimento puberal ${ }^{3,27,38}$.

Os resultados aqui obtidos demonstram uma significante redução do ângulo ANB para os dois grupos avaliados. No grupo 1, a redução média do ANB foi de $2,4^{\circ}$. O ANB do grupo 2 reduziu-se, em média, $3,2^{\circ}$. Entre os dois grupos, não foi verificada diferença significante na redução do valor médio do ANB. Esses resultados foram conseguidos pela restrição do deslocamento anterior da maxila pelos aparelhos extrabucais e pelo deslocamento anterior da mandíbula, que ocorre com o crescimento normal do paciente, durante o surto de crescimento puberal.

Os resultados obtidos, neste estudo, com o emprego de aparelhos extrabucais no tratamento de pacientes portadores de Classe II esquelética mostram que o sucesso da correção está intimamente 
relacionado com a idade do paciente e que a escotha do tipo de tração extrabucal deve ser feita de acordo com o padrão de crescimento que o paciente apresenta. Portanto, deve-se iniciar o tratamento um pouco antes do período de surto máximo de crescimento puberal, pois neste período o crescimento natural da criança contribui, juntamente com a utilização dos aparelhos extrabucais, para correção da Classe II esquelética.

\section{CONCLUSÕES}

Não houve alteração significante no padrão de crescimento facial durante o tratamento em nenhum dos grupos avaliados, independente do tipo de aparelho extrabucal utilizado. Nos pacientes do grupo 2, que possuíam tendência de crescimento vertical, o extrabucal de tração combinada mesmo não provocando efeito extrusivo sobre os molares superiores, não foi capaz de diminuir o ângulo do plano mandibular de maneira significante. $\mathrm{O}$ aumento do AFAI ocorreu de forma semelhante nos dois grupos, indicando que o aumento dessa dimensão apresenta-se diretamente relacionado ao crescimento natural, e não sofreu influência do tipo de tração extrabucal utilizada.

A maxila apresentou uma restrição no seu deslocamento anterior e verticalmente manteve-se estável. A mandíbula expressou seu crescimento e deslocou-se anteriormente de maneira similar nos dois grupos. A relação maxilomandibular apresentou uma melhora significante, com uma redução sensivel do ANB.

Enviado em: janeiro de 2006 Revisado e aceito: março de 2006

\title{
Cephalometric evaluation of anteroposterior and vertical changes in skeletal Class II patients treated with cervical or combined traction
}

\begin{abstract}
Aim: To evaluate the antero-posterior and vertical changes in patients with skeletal Class II malocclusions (ANB $>5^{\circ}$ ) by means of the cephalometry. The patients had been treated with either cervical traction device (Group 1) or combined traction device (Group 2) both in association with a Edgewise-type device. Methods: The sample consisted of 60 lateral cephalometric radiographs of 30 Caucasian individuals, 13 males and 17 females, obtained during both pre-treatment and post-treatment phases. The 15 patients of Group 1 had mean ages of 10 years and 7 months in the pre-treatment and 13 years and 9 months in the post-treatment. The other 15 patients of Group 2 had mean ages of 11 years and 5 months in the pre-treatment and 14 years and 9 months in the post-treatment. The cephalometric measures obtained in the beginning as well as in the end of the treatment were analysed and compared by using the Student's $t$ test. Results and Conclusions: No significant changes regarding the facial growth pattern during the treatment in both groups. In Group 2, whose patients had a tendency of vertical growth $\left(\mathrm{GoGn}-\mathrm{SN}>36^{\circ}\right)$, the extraoral combined traction device was not capable of reducing the mandibular plane angle despite of causing no extrusive effect on the upper molars. The anterior movement of the maxilla was restricted, whereas the vertical movement was stable in both groups studied. Although growth and anterior movement of the mandible were observed, the mandibular inclination was unchanged. The maxillary-mandibular relationship was shown to be significantly improved in both groups, with a perceptible reduction of the ANB measure.
\end{abstract}

Key words: Skeletal Class II. Cervical and combined head gear appliance. Orthodontic appliance.

\section{REFERÊNCIAS}

1. BOECLER, P. R. et al. Skeletal changes associated with extraoral appliance therapy: an evaluation of 200 consecutively treated cases. Angle Orthod, Appleton, v. 59, no. 4, p. 263-270, Apr. 1989.

2. SILVA FILHO, O. G. et al. Prevalência de oclusão normal e má-oclusão na dentadura mista em escolares da cidade de Bauru (São Paulo). Rev Assoc Paul Cir Dent, São Paulo, v. 43, p. 287-290, 1989.
3. LIMA FILHO, R. M. A. Estudo longitudinal das alterações anteroposteriores e verticais em pacientes Classe II esquelética, tratados com aparelho extra-oral de Kloehn. 2001. $102 \mathrm{f}$. Tese (Mestrado em Odontologia-Ortodontia)-Faculdade de Odontologia, Universidade Federal do Rio de Janeiro, Rio de Janeiro, 2001.

4. MAYS, R. A. A cephalometric comparison of two types of extraoral appliance used with the edgewise mechanism. Am J Orthod, St. Louis, v. 55, no. 2, p. 195-196, Feb. 1969. 
5. O'REILLY, M. T. et al. Cervical and oblique headgear: a comparison of treatment effects. Am J Orthod Dentofacial Orthop, St. Louis, v. 103, no. 6, p. 504-509, June 1993.

6. ARMSTRONG, M. M. Controlling the magnitude, direction and duration of extraoral force. Am J Orthod, St. Louis, v. 59, no. 3, p. 217-243, Mar. 1971

7. JACOBSON, A. A. A Key to the understanding of extraoral forces. Am J Orthod, St. Louis, v. 75, no. 4, p. 361-387, Apr. 1979

8. TEUSCHER, U. An appraisal of growth an reaction to extraoral anchorage. Am J Orthod, St. Louis, v. 89, no. 2, p. 113-121, Feb. 1986

9. BAUMRIND, S. E. et al. Mandibular plane changes during maxillary retraction. Am J Orthod, St. Louis, v. 74, no. 1, p. 32-40, July 1978.

10. VILELLA, O. V. Manual de cefalometria. Rio de Janeiro: Guanabara Koogan, 1998.

11. McNAMARA JR., A. Components of Class II malocclusion in children 8-10 years of age. Angle Orthod, Appleton, v. 51, no. 3, p. 177-202, July 1981.

12. LINDEN, F. P. G. M. van der. Ortodontia: desenvolvimento da dentição. Trad. de Francisco Ajalmar Maia. S. Paulo: Quintessence, 1986

13. ROSENBLUM, R. E. Class II malocclusion: mandibular retrusion of maxillary protrusion? Angle Orthod, Appleton, v. 65, no. 1, p. 49-62, Jan. 1995

14. RIESMEIJER, M. A. et al. A comparison of craniofacial Class I and Class II growth patterns. Am J Orthod Dentofacial Orthop, St. Louis, v. 125, no. 4, p. 463-471, Apr. 2004.

15. TELLES, C. S. A cefalometria aplicada à Classe II $1^{\text {a }}$ Divisão. Boletim Científico da S. B. O., São Paulo, n. 4, p. 17-22, 1978.

16. VARGERVIK, K.; HARVOLD, E. P. Response to activator treatment in Class II malocclusions. Am J Orthod, St. Louis, v. 88, no. 3, p. 242-251, Sept. 1985.

17. DALE, J. Direcionamento interceptativo da oclusão com ênfase no diagnóstico. In: GRABER, T. M.; VANARSDALL, J. R. Ortodontia: princípios e técnicas atuais. 2. ed. Rio de Janeiro: Guanabara Koogan, 1994. cap. 6, p. 264-346.

18. PROFFIT, W. R.; FIELDS, H. W. Contemporary orthodontics. 3 rd ed. St. Louis: Mosby, 2000.

19. STEINER, C. C. Cephalometrics for you and me. Am J Orthod, St. Louis, v. 39, no. 10, p. 729-755, Oct. 1953.

20. HELLMAN, M. A preliminary study in development as effect the human face. Dental Cosmos, Philadelphia, v. 69, no. 3, p. 250269, Mar. 1927.

21. DOWNS, W. B. Variations in facial relationships: their significance in treatment and prognosis. Am J Orthod, St. Louis, v. 34 , no. 10 , p. $812-840$, Oct. 1948

22. SCHUDY, F. F. The rotation of the mandible resulting from growth: its implications in Orthodontic treatment. Angle Orthod, Appleton, v. 35, no. 1, p. 36-50, Jan. 1965

23. VALE, D. M. V. do; MARTINS, D. R. Avaliação cefalométrica das estruturas dento-esqueléticas em jovens portadores de Classe II, divisão 1, brasileiros, leucodermas e de origem mediterrânea. Ortodontia, São Paulo, v. 20, n. 1/2, p. 5-17, jan./dez.1987.

24. ENLOW, D. H.; HANS, M. G. Essentials of facial growth. Philadelphia: W. B. Saunders, 1996.

25. HUBBARD, G. W. et al. A cephalometric evaluation of nonextraction cervical headgear treatment in Class II malocclusions. Angle Orthod, Appleton, v. 64, no. 5, p. 359-370, 1994.

26. RICKETTS, R. M. The influence of orthodontic treatment on facial growth and development. Angle Orthod, Appleton, v. 30, no. 3, p. 103-33, July 1960.

27. GANDINI JUNIOR, L. G. et al. Avaliação cefalométrica do tratamento da Classe II, divisão $1^{a}$, com aparelho extrabucal de Kloehn e aparelho fixo - alterações esqueléticas (parte I ). R Dental Press Ortodon Ortoped Maxilar, Maringá, v. 2, n. 6, p. 75-87, nov./dez. 1997

28. HENRIQUES, J. F. C. Estudo cefalométrico da ação da ancoragem extrabucal cervical, na dentadura mista, sobre a maxila, mandíbula e dentes, em pacientes com Classe II, divisão 1. 1979. 71 f. Dissertação (Mestrado)-Faculdade de Odontologia, Universidade de São Paulo, Bauru, 1979
29. ATTA, J. Y e et al. Estudo cefalométrico do efeito do tratamento ortodôntico sobre a mandíbula em pacientes com Classe II divisão 1. Rev Odontol USP, São Paulo, v. 4, n. 1, p. 43-48, jan./mar. 1990

30. VADEN, J. L. et al. Clinical ramifications of posterior and anterior facial height changes between treated and untreated Class II samples. Am J Orthod Dentofacial Orthop, St. Louis, v. 105 no. 5, p. 438-443, May 1994

31. COOK, A. H. et al. A control of the vertical dimension in class II correction using a cervical headgear and lower utility arch in growing patients. Part I. Am J Orthod Dentofacial Orthop, St. Louis, v. 106, no. 4, p. 376-388, Oct. 1994.

32. BURKE, M.; JACOBSON, A. Vertical changes in high-angle Class II division 1 patients treated with cervical or occipital pull headgear. Am J Orthod Dentofacial Orthop, St. Louis, v. 102, no. 6 p. 501-508, Dec. 1992

33. KLEIN, P. L. An evaluation of cervical traction on maxilla and the upper first permanent molar. Angle Orthod, Appleton, v. 27 no. 2, p. 61-68, Jan. 1957

34. STÖCKLI, P. W.; TEUSCHER, U. M. Ortopedia combinada com ativador e extrabucal. In: GRABER, T. M.; VANARSDALL JÚNIOR, R. L. Ortodontia: princípios e técnicas atuais. 2. ed. Rio de Janeiro: Guanabara Koogan, 1994. cap. 8, p. 400-465.

35. KLOEHN, S. J. Guiding alveolar growth and eruption of teeth to reduce treatment time and produce a more balanced denture and face. Angle Orthod, Appleton, v. 17, no. 1, p. 10-33, Jan./ Apr. 1947.

36. BRODIE, A. G. The fourth dimension in Orthodontia. Angle Orthod, Appleton, v. 24, no. 1, p. 15-30, Jan. 1954

37. SEIXAS, M. R. Efeito do aparelho extra-oral de Kloehn na maxila e primeiros molares permanentes superiores no tratamento da Classe II, $\mathbf{1}^{\text {a }}$ divisão de Angle. 1998. 106 f. Tese (Mestrado em Odontologia-Ortodontia)-Faculdade de Odontologia, Universidade Federal do Rio de Janeiro, Rio de Janeiro 1998.

38. POULTON, D. R. The influence of extraoral traction. Am J Orthod, St. Louis, v. 53, no. 1, p. 8-18, Jan. 1967

39. TELLES,C. H. C. Avaliação cefalométrica dos efeitos do extraoral de Kloehn em pacientes submetidos a tratamento ortodôntico. 1982. 95 f. Tese (Mestrado em -Ortodontia)-Faculdade de Odontologia, Universidade Federal do Rio de Janeiro, Rio de Janeiro, 1982

40. CANGIALOSI, T. J. et al. A cephalometric appraisal of edgewise Class II nonextraction treatment with extraoral force. Am J Orthod Dentofacial Orthop, St. Louis, v. 93, no. 4, p. 315-324, Apr. 1988.

41. KNIGHT, H. The effects of three methods of orthodontic appliance therapy on some commonly used cephalometric angular variables. Am J Orthod Dentofacial Orthop, St. Louis, v. 93 p. 237-244, 1988.

42. HENRIQUES, J. F. C. Estudo cefalométrico comparativo de três tipos de ancoragem extrabucal, sobre as estruturas dentoesqueléticas, em pacientes com Classe II, $1^{\text {a }}$ divisão. 1993. 166 f. Tese (Livre-Docência)-Faculdade de Odontologia, Universidade de São Paulo, Bauru, 1993.

Endereço para correspondência

Márlio Vinícius de Oliveira

Rua Mato Grosso, 560 - Universal

CEP: 32.686-050 - Betim/MG

E-mail: marlio_vinicius@ig.com.br 